ARTICLE

DOI: $10.1038 / s 41467-018-05612-8$

\title{
A sulfotransferase dosage-dependently regulates mouthpart polyphenism in the nematode Pristionchus pacificus
}

\author{
Linh T. Bui (i) ${ }^{1}$, Nicholas A. Ivers ${ }^{1} \&$ Erik J. Ragsdale (iD ${ }^{1}$
}

Polyphenism, the extreme form of developmental plasticity, is the ability of a genotype to produce discrete morphologies matched to alternative environments. Because polyphenism is likely to be under switch-like molecular control, a comparative genetic approach could reveal the molecular targets of plasticity evolution. Here we report that the lineage-specific sulfotransferase SEUD-1, which responds to environmental cues, dosage-dependently regulates polyphenism of mouthparts in the nematode Pristionchus pacificus. SEUD-1 is expressed in cells producing dimorphic morphologies, thereby integrating an intercellular signalling mechanism at its ultimate target. Additionally, multiple alterations of seud-1 support it as a potential target for plasticity evolution. First, a recent duplication of seud-1 in a sister species reveals a direct correlation between genomic dosage and polyphenism threshold. Second, inbreeding to produce divergent polyphenism thresholds resulted in changes in transcriptional dosage of seud-1. Our study thus offers a genetic explanation for how plastic responses evolve.

\footnotetext{
${ }^{1}$ Department of Biology, Indiana University, 915 E. 3rd St., Bloomington, IN 47405, USA. These authors contributed equally: Linh T. Bui, Nicholas A. Ivers. Correspondence and requests for materials should be addressed to E.J.R. (email: ragsdale@indiana.edu)
} 
D evelopmental plasticity, the ability to produce multiple phenotypes from a single genotype, is a ubiquitous feature of multicellular life. At its extreme, plasticity takes the form of polyphenism, by which discrete alternative morphs develop, often with disparate ecological roles and sometimes exhibiting evolutionary novelties ${ }^{1,2}$. Because its binary outputs imply a limited number of factors needed for a switch mechanism, polyphenism offers a tractable model for studying how plasticity is controlled and responds to selective pressures. Genetic regulators of morphological polyphenism have indeedbegun to be revealed ${ }^{3-6}$, and the selectable variation shown for polyphenisms ${ }^{7,8}$ suggests that genetic changes in the control of polyphenism may ultimately be identified. However, the genetic architecture of polyphenism is still not well understood, and it has remained elusive how such architecture should change across species with different plastic responses under different ecological pressures.

The omnivorous nematode Pristionchus pacificus has a polyphenism in its adult mouthparts, allowing individuals to take different diets in response to local environmental cues experienced as postembryonic larvae (Fig. 1). Specifically, the nematodes develop either into a "stenostomatous" (St) morph, which has a narrow mouth specialised for feeding on microbes, or into a "eurystomatous" (Eu) morph with opposing, moveable teeth that allow a broader diet, including predation on other nematodes 9 . Production of the Eu morph, which confers a fitness benefit over the St morph when deprived of bacteria and offered nematodes as prey $^{9}$, is influenced by the local availability of microbial food as sensed by starvation ${ }^{10}$ and local crowding by conspecifics ${ }^{11}$. How responsive (i.e., plastic) the polyphenism decision is to environmental changes varies across populations and species ${ }^{3,12}$-for example, the laboratory reference strain of $P$. pacificus is mostly (90\%) Eu even when well-fed under laboratory conditions-such that the set point of the polyphenism threshold can vary, potentially offering more or less sensitivity to given cues according to local environmental pressures. At a genetic level, this polyphenism is regulated by the lineage-specific, dosage-dependent sulfatase EUD- ${ }^{3}$. This completely penetrant regulator channels pheromone signalling ${ }^{13}$, endocrine (DAF-12dafachronic acid) signalling ${ }^{10}$, and information from chromatin modifiers and antisense RNAs ${ }^{14}$ into a single switch. EUD-1 activity depends on the nuclear receptor NHR-40, which was proposed to be at the transcriptional terminus of the switch mechanism ${ }^{15}$. Yet despite an emerging genetic knowledge of this polyphenism and of plasticity in general, how genetic changes result in the evolution of plastic responses is little understood. Here, through the identification of a new polyphenism switchgene, we show that a plastic response (i.e., morph ratio) is decided by the relative dosage of putative signal-modifying enzymes. Specifically, we have discovered a sulfotransferase with dosage-dependent epistasis over EUD-1 to set a threshold for a plasticity switch. Furthermore, we show that environmental, laboratory-selected, and interspecies changes in plasticity correlate with changes in the relative dosage of these genes. Our findings thus provide genetic insight into how the molecular regulation of polyphenism evolves to produce new plastic responses.

\section{Results}

Several recessive mutants suppress a polyphenism switch gene. As an unbiased approach to identify genes making up a polyphenism switch, we conducted a forward genetic screen for recessive suppressors of the mutant strain eud-1(tu445), which is completely St (i.e., Eud, eurystomatous-form-defective). We mutagenised eud-1(tu445) mothers, isolated their putatively
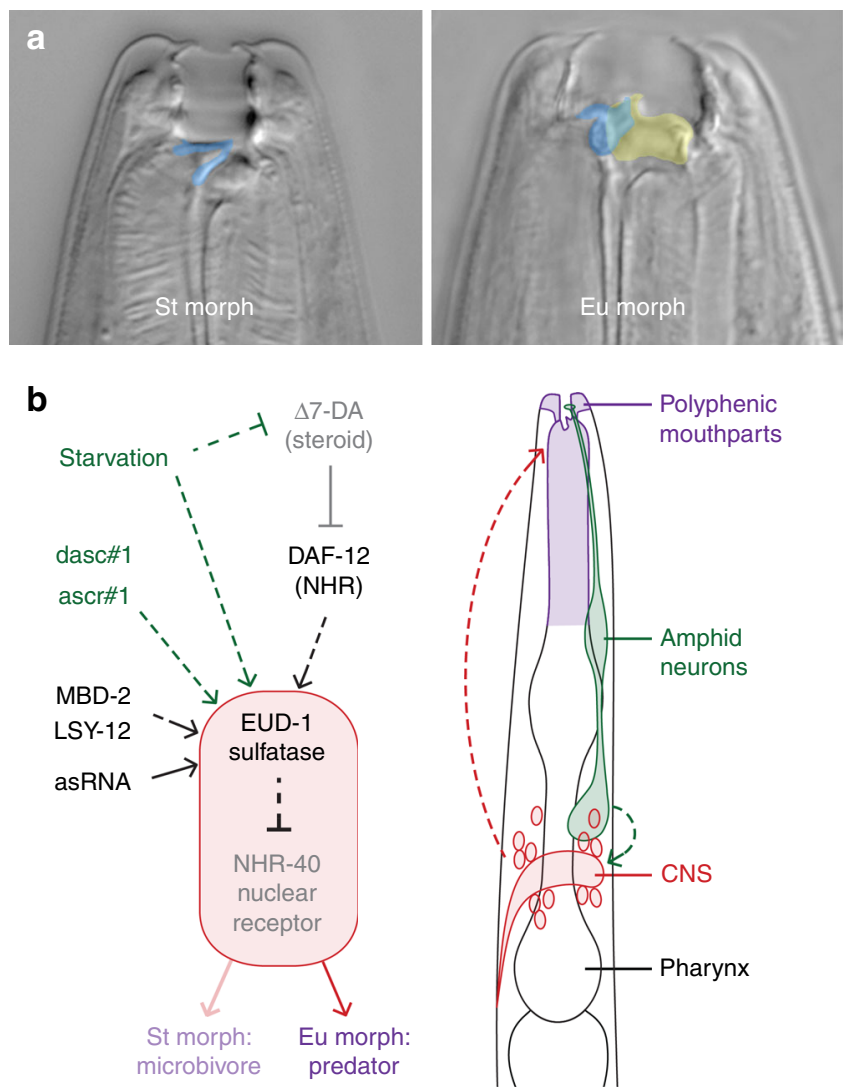

Fig. 1 Developmental regulation of polyphenism in Pristionchus pacificus. a Alternative morphs (eurystomatous, "Eu," and stenostomatous, "St") have mouthparts that allow different ecological roles: the Eu morph differs in the shape of its dorsal tooth (false-coloured blue) and the presence of an additional, opposing tooth (yellow), which enable predatory feeding on other nematodes. b Known genetic factors regulating the mouth-form polyphenism (left) and their known or presumed spatial context in the developing larva (right). Sensitivity to environmental cues (green) of nutritional stress and crowding (pheromones dasc\#1 and ascr\#1) occurs during postembryonic development, where "starvation" (experimental withholding of food) has an influence as early as the first post-hatch (J2) stage $^{10}$ and the presence of other nematodes as late as the $\mathrm{J} 3$ and possibly J4 (pre-adult) stage ${ }^{11}$. Downstream of these cues, which in C. elegans are primarily intercepted and interpreted by several amphid neurons ${ }^{55,56}$, parallel regulatory elements including endocrine (dafachronic acid) signalling, chromatin modification (by LSY-12 and MBD-2), and eud-1 asRNAs converge on a polyphenism "switch." This switch includes EUD-1 and NHR-40, which together comprise a signalling system that originates in the central nervous system (red) and ultimately decides between alternative feeding morphologies (purple). The decision is irreversible by the J4-adult moult, resulting in adult phenotypes best matched to the environment experienced as larvae

heterozygous $F_{1}$, and screened segregant $F_{2}$ for mutants showing an Eu phenotype. From a screen of $\sim 10,300$ haploid genomes, we identified seven recessive mutants. These mutants fell into one of three complementation groups, suggesting that the polyphenism switch comprises, and can feasibly be described from, a finite number of factors with non-deleterious mutant phenotypes. One of the complementation groups consisted of two mutant alleles, $\sup (i u b 7)$ and $\sup (i u b 8)$, which were fully penetrant for their constitutive suppression of the Eud phenotype, even under wellfed conditions (Fig. 2a; Supplementary Table 1). We named the gene represented by these alleles seud-1 (suppressor-of-eud-1). 
a

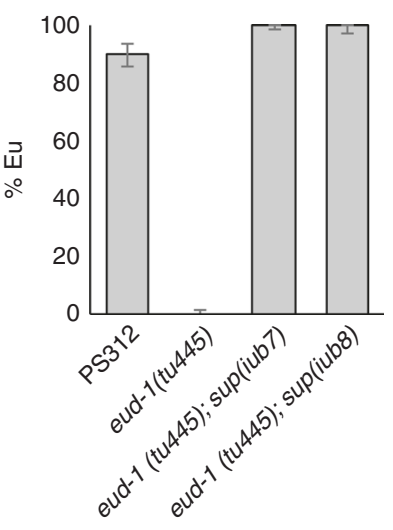

C

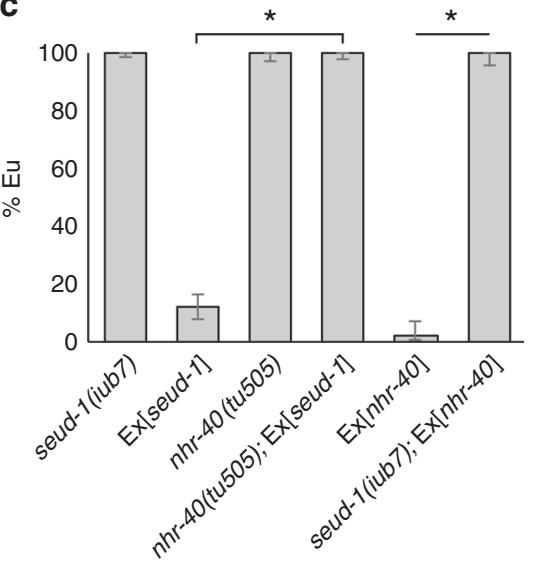

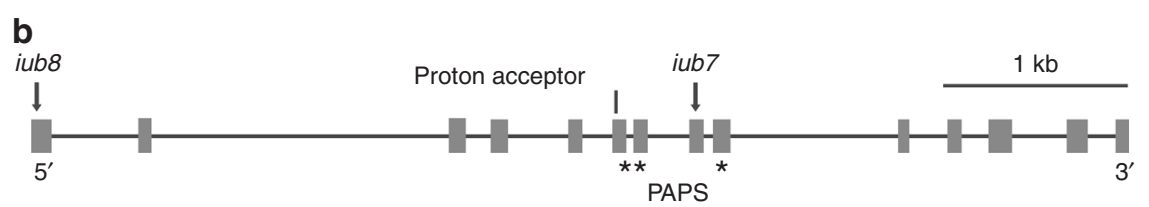

Fig. 2 Mutants, gene structure, and epistasis of the plasticity regulator seud-1. a In contrast to the wild-type and eud-1 phenotypes, mutants iub7 and iub8 are completely penetrant for the suppressor-of-Eud (and all-eurystomatous) phenotype. b Mutants uncovered were the null alleles iub7 and iub8, both of which lack an incomplete sulfotransferase domain (catalytic proton acceptor + PAPS sulfate donor). c Mutant rescue of seud-1 mutants with a transgenic construct, Ex[seud-1], indicates the dosage-dependent activity of this gene, whose function is reciprocally necessary with nhr-40 for the polyphenism switch. Phenotype is the proportion of eurystomatous (Eu) individuals produced per clone. Scale bar, $20 \mu \mathrm{m}$. \% Eu, proportion of eurystomatous nematodes. ${ }^{\star} P<0.0001\left(\chi^{2}\right.$-test). Whiskers represent a $95 \%$ confidence interval

A sulfatase is suppressed by a sulfotransferase. To identify the locus of seud-1(iub7) and seud-1(iub8), we backcrossed each mutant and resequenced its genome. We scanned all annotated genes for non-identical and potentially harmful (nonsense, missense, and splice-site) mutations in both mutants. The entire genome yielded only one predicted gene, Contig20-aug8366.t1, with harmful lesions unique to each mutant. This gene, located on Chromosome I, encodes a cytosolic sulfotransferase and is one of several P. pacificus homologues of Caenorhabditis elegans ssu-1. In C. elegans, SSU-1 functions in dauer diapause, another type of environmentally conditioned development, acting with the steroid hormone receptor DAF-12/VDR in ASJ amphid neurons to suppress formation of dauer downstream of insulin (DAF-2) signalling ${ }^{16,17}$. Mutant lesions in $i u b 7$ and $i u b 8$ were nonsense mutations in Exon 8 and Exon 1, respectively, of the gene as annotated from expressed RNAs (Fig. 2b). The iub8 lesion lies upstream of sequences encoding a proton acceptor $\left(\mathrm{H}^{164}\right)$ for a substrate-binding domain, and $i u b 7$ lies within a sulfate-donor (3'-phosphoadenosine-5' -phosphosulfate, PAPS) domain (Supplementary Table 2). Sequences for both functional domains are highly conserved across nematodes and the mammalian homologue SULT2A1 (Supplementary Fig. 1), suggesting the conserved function of SEUD-1 as a cytosolic sulfotransferase in $P$. pacificus.

SEUD-1 is a genetic intermediary between EUD-1 and NHR40. Given the likely function of SSU-1 as a sulfotransferase, we hypothesised that this enzyme may act, like the sulfatase EUD-1, on a substrate upstream of NHR-40. To test this idea, we investigated its epistasis with $n h r-40$, particularly using a line of the null mutant seud-1(iub7) that was outcrossed to restore wild-type alleles at eud-1 (Supplementary Table 3). Because $n h r-40$ and seud-1 both promote the St form in wild-type animals, we performed epistasis tests by combining loss-of-function mutations in one gene with transgenic overexpression in the other. These tests specifically used an nhr-40 mutant allele (tu505) that, like all previously recovered $P$. pacificus $n h r-40$ mutants, incurred a nonsynonymous mutation that is fully penetrant for its Eu-promoting phenotype ${ }^{15}$. First, we transgenically over-expressed a wild-type (PS312) construct of $n h r-40$ in a null seud-1(iub7) mutant. This seud-1(iub7); $\operatorname{Ex}[n h r-40]$ line resulted in the all-Eu phenotype of seud-1(iub7) rather than the mostly-St phenotype of the overexpressing line $\operatorname{Ex}[n h r-40]\left(P<10^{-4}\right.$; Fig. 2c; Supplementary Table 4), indicating that seud-1 either acts downstream of NHR40 or, alternatively, is necessary to sulfate a signal that ultimately activates NHR-40-mediated transcription. In the former model, seud-1 over-expression should suppress the phenotype of $n h r-40$ mutants, whereas in the latter model the reverse should be the case. To distinguish between these possibilities, we transgenically over-expressed a wild-type genomic clone of seud-1, including its presumptive $5^{\prime}$ and $3^{\prime}$ regulatory elements, in nhr-40(tu505) mutants (Supplementary Table 5). Although transgenic Ex[seud1] animals wild-type for $n h r-40$ were mostly St, Ex[seud-1] animals with loss-of-function $n h r-40$ alleles exhibited the $n h r-40$ mutant phenotype, rather than the seud-1 over-expression phenotype $\left(P<10^{-4}\right.$; Fig. 2c; Supplementary Table 4). Therefore, reciprocal epistasis tests indicate that SEUD-1 acts between EUD1 and NHR-40 and has an enzymatic function necessary for NHR-40 function.

SEUD-1 is expressed at the site of the polyphenism. Because expression of eud-1 and $n h r-40$ does not overlap, and because $n h r-40$ is pleiotropically expressed across several tissues ${ }^{15}$, the anatomical scope of the polyphenism switch was previously unknown. We therefore studied the expression of seud-1 to reconstruct the switch in space. To localise expression of seud-1, we generated a transcriptional, nucleus-localised, fluorescent (RFP) reporter using the same promoter sequence that was sufficient to over-express seud-1. This transgenic construct reported seud-1 expression in the cells making up the anterior body wall (face), stoma (mouth), and anterior pharynx, which have 

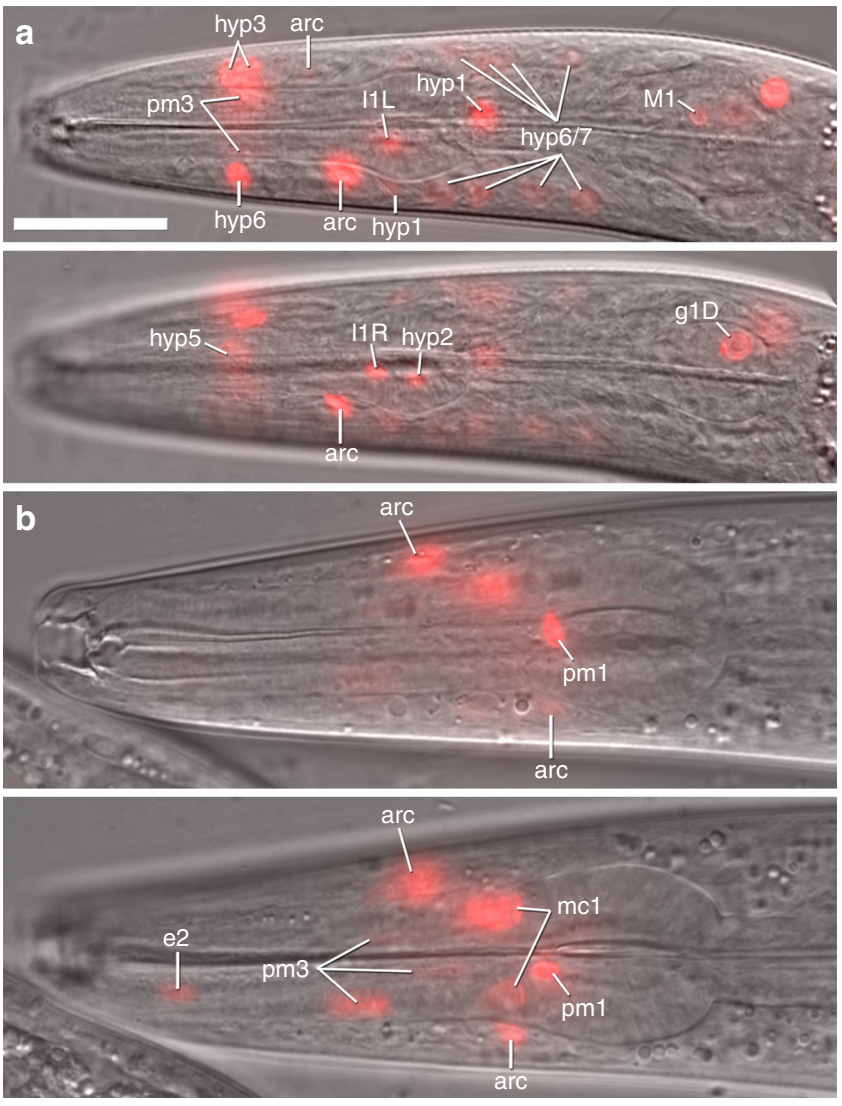

Fig. 3 Spatial expression of seud-1 in Pristionchus pacificus. Expression of a nucleus-localised seud-promoter::TurboRFP reporter is shown for (a) a J3 larva and for (b) a stenostomatous adult hermaphrodite shortly after the final moult, each in two focal planes. $\mathrm{J} 3$ is shown in sagittal and left of sagittal planes; adult, in sagittal and right of sagittal planes. Expression is reported for pharyngeal myoepithelial (pm1, pm3, pm4), epithelial (e2, $\mathrm{mc1}$ ), dorsal gland (g1D), tooth cell-innervating neurons (11, M1), interfacial (posterior and anterior arcade, "arc"), and anterior epidermal (hyp) cells of, collectively, the anterior pharynx, stoma (mouth), and anterior body wall. Not all expressing cells, some of which (e.g., hyp, arcade cells) could not reliably assigned to a syncytium, are labelled. Expression ontogeny across life-stages suggests variation in timing among various components of dimorphic mouth morphologies, although expression consistently encompassed the breadth of morphology described or, in the case of pharyngeal neurons, presumed to be dimorphic in P. pacificus. Scale bar (for all images), $20 \mu \mathrm{m}$

homologues in $C$. elegans ${ }^{18}$ and which together produce the feeding morphology that is dimorphic in P. pacificus (Fig. 3). We detected expression in these cells throughout postembryonic juvenile stages (J1-J4), dauer (a facultative, third-stage diapause larva), and young adults (Supplementary Fig. 2). Expression was consistently most pervasive across cell classes at the J2 and J3 stages (Fig. 3a), thereafter tapering in the J4 and early adult stages (Fig. 3b), and indeed at the adult stage, when morphs could be distinguished, no obvious qualitative differences in expression between Eu and St morphs were observed. Additionally, although individuals of a given life-stage varied in which particular cells were expressed when the animals were caught for examination, all larval stages were collectively shown to express seud-1 in this set of cells. These findings suggest that this polyphenism switch factor is active in dimorphic tissue throughout postembryonic development, including diapause, until the polyphenism is expressed at the adult stage.
The specific cells expressing the reporter were those making up the anterior epidermal (hyp) syncytia, which together comprise the anterior body wall and secrete the outer lining of the mouth (cheilostom) $)^{19}$, the region of the mouth and head that is dimorphic in width; the anterior and posterior arcade cells, which join the mouth opening to the pharyn $\mathrm{x}^{20}$; epithelial cells e2 and $\mathrm{mc1}$, and myoepithelial cells pm1, pm3, and pm4, which together comprise the pharyngeal muscle and secrete the extracellular teeth ${ }^{21}$. In addition to cells in the mouth and anterior pharynx, strong expression was detected at early juvenile (J2 and J3) stages in the pharyngeal gland (g1) cell, the nuclei of which are in the pharyngeal basal bulb (Fig. 3a). This cell comprises a gland whose duct terminates in the dorsal tooth, the secretions of which have been hypothesised to be involved in predatory feeding and which is, compared with C. elegans, highly innervated by the pharyngeal nervous system ${ }^{18}$. Other cell classes reporting seud-1 were M1, I1, and possibly I2 (Fig. 3a), all of which innervate pm1, the cell actuating the dorsal tooth, and are thus hypothesised to reflect dimorphism in pharyngeal behaviour $^{18}$. Because the cells reporting seud-1 overlap with several cells in which $n h r-40$ is expressed ${ }^{15}$, seud-1 and $n h r-40$ likely control the polyphenism decision at the site of the morphological dimorphism. Together, these results indicate that seud-1 is expressed at the terminal integration site of the polyphenism pathway in $P$. pacificus, whereas EUD-1 regulates signalling upstream of these target cells.

Polyphenism is set by dosages of enzymes of opposed function. Because transgenic over-expression of seud-1 showed the gene to be dosage-dependent, we hypothesised that the relative dosages of seud-1 and eud-1 may together establish the threshold for the polyphenism switch. To test this idea, we manipulated wild-type copy number, a feature shown to affect expressivity in eud-1 hemizygotes and heterozygous mutants ${ }^{3}$. Specifically, we used eud-1 and seud-1 mutants to generate all possible combinations of homozygotes, heterozygotes, and hemizygotes, from both cross directions, for both genes (Supplementary Table 6). As a result, relative copy number of functional eud-1 and seud-1 alleles was sufficient to gradate the ratio of morphs among the $\mathrm{F}_{1}$ from all-Eu to all-St (Fig. 4a). All genotypes with one copy of each gene restored the phenotype of wild-type hermaphrodites (morphological females), which have two copies of each, indicating the relative dosage dependence of eud-1 and seud-1. Moreover, animals with one copy of seud-1 but two copies of eud-1 were almost all-Eu, as the Eu-promoting eud-1 is favoured, although a few animals were still St. Lastly, no number of copies of eud-1 could rescue Eu formation in the absence of wild-type seud-1. Together, these results indicate that, under a given environmental regimen, relative dosage of the two genes set the threshold for the mouthmorph ratio of $P$. pacificus.

Morph-inducing cues differentially regulate seud-1. Given the dependence of the morph ratio on relative genomic dosage of eud-1 and seud-1, we investigated whether transcriptional dosage of these genes reflected differences in morph ratio induced under different environments. Namely, we predicted that the different morph-inducing cues would result in higher eud-1:seud-1 transcript ratios in an Eu-inducing compared with a St-inducing environment. To test this prediction, we performed an analysis of RNA transcripts produced across multiple nutritive environments demonstrated to elicit different plastic responses in P. pacificus ${ }^{22}$. Because the only variable in this experiment was food source, we interpreted any differences in phenotype and gene expression to be the result of dietary cues. Results of this analysis showed that nematodes cultured on a relatively St-inducing diet of 

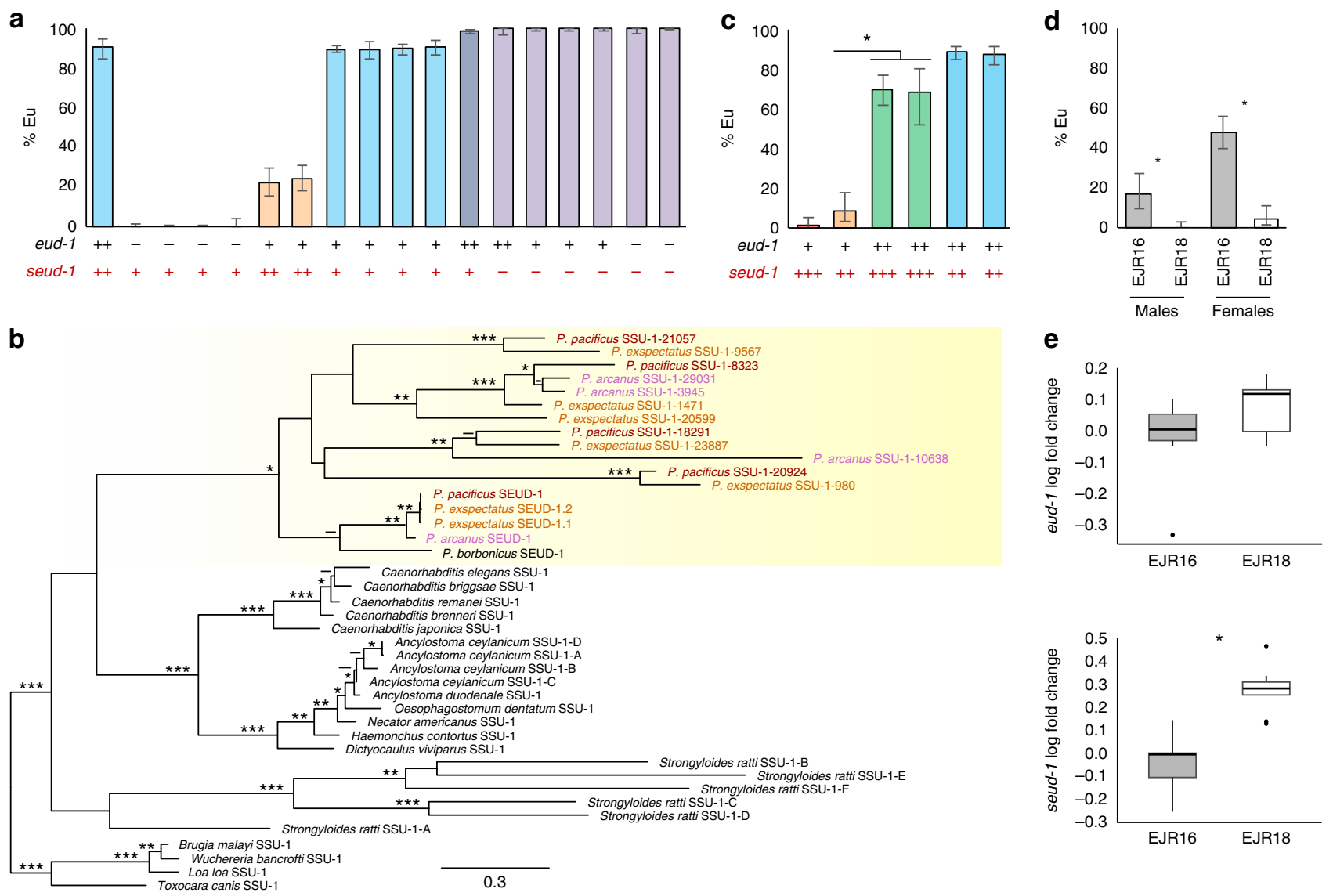

Fig. 4 Genomic dosage, duplication, macroevolution, and inbred divergence of seud-1. a Compared to the wild-type (left bar), animals with the relative dosage of eud-1 to seud-1 manipulated (each dosage ratio marked by a unique bar colour) show a gradation of plasticity thresholds, as measured by the percentage of eurystomatous (Eu) individuals, produced in a common environment. Specific crosses used to produce dosages of eud-1 to seud-1, as indicated along the $x$-axis, are given in Supplementary Table 6. Each plus-sign below chart represents a functional (wild-type) gene copy present in cross offspring; each minus-sign, a non-functional (mutant) copy. b seud-1 (boldface font) is the product of gene duplications, particularly a large radiation of sulfotransferase-encoding ssu- 1 homologues, specific to a lineage including Pristionchus (yellow shading). Divergences even between three closely related species (marked in differently coloured fonts) indicates the dynamic divergence of these homologues, including the recent duplication of seud-1 in $P$. exspectatus. Amino-acid sequences were analysed by maximum likelihood. Bootstrap support values: ${ }^{\star \star \star} 99-100 \%$; ${ }^{\star \star} 96-98 \%$; ${ }^{\star 75}-95 \%$; $-50-74 \%$. c Manipulation of competing (eud-1:seud-1, each dosage ratio marked by a unique bar colour) gene dosages using hybrids with $P$. exspectatus, support the function of both duplicates and their consequences on the different plasticity phenotype observed for $P$. exspectatus. Specific crosses used to produce dosages of eud-1 to seud-1, as indicated along the $x$-axis, are given in Supplementary Table 9. ${ }^{\star} P<0.0001$ ( $\chi^{2}$-test). d Lines of $P$. exspectatus (EJR16, EJR18) were systematically inbred from a heterozygous parent strain to result in different polyphenism ratios. ${ }^{\star} P<10^{-4}$ ( $\chi^{2}$-test). e Inbred lines of $P$. exspectatus diverged in transcriptional dosage ( $\log _{2}$ fold change of expression) of seud-1 (i.e., Pex-seud-1.1 + Pex-seud-1.2), in particular relative to eud-1, expression of which did not change $(P>0.05)$. Box plots show $\Delta \Delta$ Ct values (centre line, median; box limits, upper and lower quartiles; whiskers, $1.5 \times$ interquartile range; points, outliers). ${ }^{\star} P<0.001$ (Tukey's test). Whiskers represent a $95 \%$ confidence interval

\begin{tabular}{|c|c|c|c|c|c|}
\hline $\begin{array}{l}\text { Food } \\
\text { source }\end{array}$ & $\begin{array}{l}\% \\
\text { Eu }\end{array}$ & $\begin{array}{l}\text { seud-1 } \log _{2} \\
\text { FC }\end{array}$ & $p$-value & $\begin{array}{l}\text { eud-1 } \log _{2} \\
\text { FC }\end{array}$ & $p$-value \\
\hline OP50 & 89 & - & - & - & - \\
\hline $\begin{array}{l}\text { C. } \\
\text { curvatus }\end{array}$ & 84 & 0.4338 & 0.186 & 0.7282 & 0.0269 \\
\hline C. albidus & 50 & 2.1083 & $1.27 \mathrm{E}-12$ & 0.7725 & 0.00250 \\
\hline
\end{tabular}

Log fold change ( $\log _{2}$ FC) of seud-1 and eud- 1 is shown for Pristionchus pacificus (strain PS312) grown under different morph-inducing environments (Cryptococcus yeast species offered as food) compared to a diet E. coli OP50. \% Eu is the median proportion of the eurystomatous (Eu) morph out of total individuals screened, as previously reported on those diets $22 . p$-values were obtained using an exact test with a Benjamini-Hochberg correction for multiple comparisons

Cryptococcus albicus yeasts resulted in higher enrichment of seud1 expression compared with a diet of E. coli OP50 (Table 1). In contrast, a relatively Eu-inducing diet of C. curvatus yeasts showed no difference in either phenotype or seud-1 expression. Further, eud-1 transcript levels were lower on a diet of C. albicus, and only slightly lower in C. curvatus, further shifting the ratio of eud-1 to seud-1 transcripts on the former diet. Our results thus indicate that, in a single genetic background, different morphinducing environmental cues result in relative transcriptional dosage of opposing factors making up the polyphenism switch.

seud-1 homologues are dynamically radiating in Pristionchus. To reconstruct the evolutionary origin of seud-1, we performed a phylogenetic analysis of seud-1 and other identifiable homologues of C. elegans ssu-1 in Pristionchus and outgroups (Supplementary Table 7). This analysis showed that three sibling species of Pristionchus each had at least four to seven homologues of $s s u-1$. Several of these genes have high inferred divergence and dubious orthology (Fig. 4b), despite the species' being close enough to interbreed $^{23}$. In contrast, the most recent common ancestor of Pristionchus and outgroups likely carried a single ssu-1 
homologue. Therefore, a radiation of $s s u-1$ duplicates has been specific to a lineage including Pristionchus and coincides with the presence of polyphenism in evolutionary history ${ }^{12}$. A product of this radiation was seud-1, for which orthology could be established across Pristionchus species (Fig. 4b). However, even within this clade, a recent duplication of seud-1 was detected, specifically in P. exspectatus. Pairwise counts of $d_{\mathrm{N}} / d_{\mathrm{S}}$ between the two Pexseud-1 duplicates (0.027), as well as between each copy and the reference allele for $P$. pacificus $(0.012,0.011)$, suggest that both are under strong purifying selection (i.e., $d_{\mathrm{N}} / d_{\mathrm{S}} \ll 1.0$ ) rather than harbouring an incipient pseudogene. Furthermore, the coding sequences of the two copies are $>99 \%$ identical at the amino-acid level, suggesting a similar enzymatic function between duplicates.

Gene duplication correlates with plasticity changes among species. Because the $P$. pacificus polyphenism is sensitive to genomic dosage of switch genes, we hypothesised that the recent duplication of seud-1 in P. exspectatus has changed the species' plasticity phenotype. The sampled strain of this species, prior to its laboratory inbreeding to an all-St phenotype, produced more St animals than most strains of $P$. pacificus under laboratory conditions ${ }^{3}$. In principle, duplication could provide an instant change in a plasticity response given the standing trans-regulation of factors making up a polyphenism switch: in P. exspectatus, this would result in the St morph being more likely to develop in a given environment. We tested whether Pex-seud-1.1 and Pexseud-1.2, which are both expressed (Supplementary Table 8), had a combined influence on the polyphenism decision, using $P$. pacificus mutants to create interspecific hybrids with different relative copy numbers of eud-1 and seud-1 (Supplementary Table 9). By examining $F_{1}$ females from these crosses, we could standardise the regulatory genetic background of these genotypes, which has likely diverged in addition to gene dosage. As predicted, changing the ratio of copies of eud-1 to seud-1 (1:3, 1:2, $2: 3$, and 2:2) in hybrids resulted in a gradation of plasticity phenotypes (Fig. 4c). In particular, hybrid phenotypes showed a difference between double "heterozygotes" (1:2) and double "homozygotes" (2:3) from either $P$. pacificus mothers $(P<0.0001$, $Z$-test $)$ or $P$. exspectatus mothers $(P<0.0001, Z$-test $)$, consistent with both Pex-seud-1 duplicates having an influence on the morph ratio in P. exspectatus. Taken together, these results show a clear correlation between the duplication of one of two opposing switch factors and a divergent plastic response (i.e., morph ratio) to a given environmental regimen.

Inbred plasticity differences correlate with seud-1 regulation. Lastly, we examined whether changes in relative transcriptional dosage could be detected among haplotypes differing in their plastic responses. First, we quantified expression of eud-1 and seud-1 in three genetically distant $P$. pacificus isolates ${ }^{24,25}$ that vary in their morph ratios in a standardised environment ${ }^{3}$. While two St-biased strains (RS5200B, RS5410), as predicted, showed lower $(P<0.05$, Tukey's test) or a trend toward lower $(P=0.1$, Tukey's test) eud-1 expression than the California strain, seud-1 expression was also lower in these St-biased strains $(P<0.05$, Tukey's test; Supplementary Fig. 3), indicating that at least some variation must be attributed to regulatory differences beyond the simple scaling of eud-1 and seud-1 expression. Furthermore, no coding-sequence variation could be found to explain this phenotypic variation, perhaps due to additional differences in other polyphenism factors (e.g., NHR-40) or their target gene repertoire. Therefore, we next performed an experiment to test whether relative dosages could be bred for divergent phenotypes on a shorter time scale, specifically from a single, heterozygous laboratory population. To do this, we systematically inbred lines of $P$. exspectatus, a gonochoristic species harbouring higher levels of heterozygosity than $P$. pacificus ${ }^{25}$, possibly including for plasticity-regulating genes. Systematic inbreeding for 10 generations indeed resulted in lines being fixed for different polyphenism thresholds in both females $\left(P<10^{-4}, \chi^{2}\right.$-test $)$ and males $(P<$ $10^{-4}, \chi^{2}$-test) in standardised environments (Fig. 4d). Furthermore, when we measured the relative expression of eud-1 and total "seud-1" (seud-1.1+seud-1.2) in these lines, we found that an increase in St animals correlated with increased relative transcription $(P<0.001$, Tukey's test $)$ of combined seud-1 duplicates (Fig. 4e). Thus, natural variation in transcriptional regulation could be selected to result in both in divergent plastic responses and in divergent switch-gene transcription, implicating these genes as potential targets for plasticity evolution.

\section{Discussion}

In summary, we describe a genetic model for a signalling system regulating developmental plasticity, and we have identified molecular targets of plasticity evolution (Fig. 5). Our findings support two major insights about plasticity regulation and its capacity to change. First, we have detailed how a pair of enzymes can fine-tune a polyphenism threshold without requiring changes to signal production itself. Although hormones have been long known to mediate morphological polyphenism in animals ${ }^{26}$, the molecular mechanisms that integrate polyphenism have been poorly understood. In $P$. pacificus, a plasticity threshold is mediated by opposing factors regulating local signalling function, particularly through sulfation and desulfation. Further, sulfation by SEUD-1 is instructive, promoting the activity of NHR-40, possibly by locally providing a metabolic precursor or modifying transport of an active ligand ${ }^{27}$. Alternatively, SEUD-1 and EUD-1 may regulate NHR-40 itself, such as through its acetylation

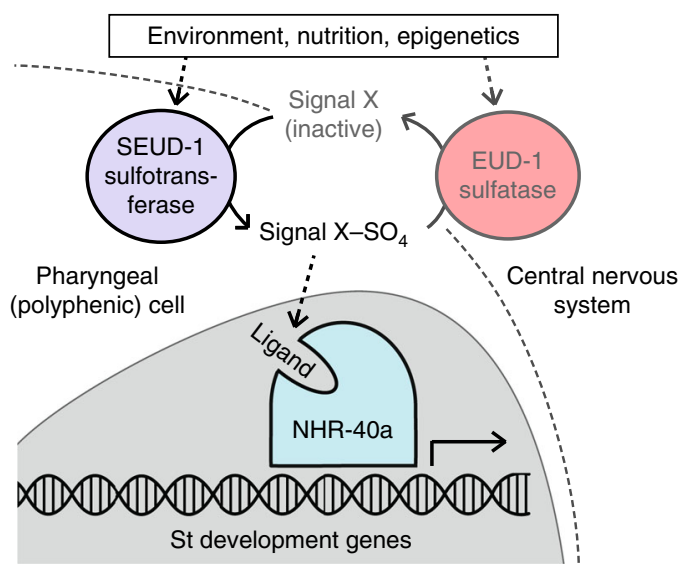

Fig. 5 A genetic model for polyphenism regulation and its potential evolutionary targets. A signalling mechanism originating in somatic neurons is integrated at the site of the polyphenism, where seud- 1 and $n h r-40$ are expressed. Results suggest a model whereby the maintenance of a sulfated signal, possibly modified extracellularly by the signal peptide-including isoform of EUD- $1^{3}$, promotes St-morph gene transcription (or, alternatively, Eu-morph gene repression) through NHR-40, particularly its complete (DNA-binding and ligand-binding) isoform (NHR-40a). Consistent with previous studies of morph-inducing cues, function, and expression of seud-1 suggests that this switch mechanism may be responsive to cues throughout postembryonic development, before making its irreversible decision at the J4-adult moult. The threshold for the morph ratio is thus set by the relative dosage of two enzymes of opposing function, such that changes to this dosage can alter the threshold for the polyphenism under a given set of environmental conditions 
state $^{28}$, although the apparent absence of EUD-1 from polyphenic tissue makes this scenario less likely. Signal modification would also be consistent with the inferred activity of C. elegans ssu-1, for which mutants show altered levels of sulfated sterols ${ }^{14}$. We therefore propose that a signalling molecule, still to be identified, is alternately modified to regulate its local activity at the targets of the polyphenism pathway.

Secondly, our study gives a genetic explanation for how genetic changes to a polyphenism mechanism produce new plasticity responses in different lineages. Opposing signalling modifiers offer an alternative evolutionary target besides the production of signals themselves, which are often pleiotropic and likely to be constrained ${ }^{29,30}$. This target also differs from a signal's ultimate receptor, to which mutations can have a dramatic impact on phenotype $^{31}$ and also be hindered by pleiotropy, as observed for NHR- $40^{32}$. Thus, the balance between competing enzymatic regulators can be shifted to change the threshold response required for a switch. In principle, changes to such a system may be fine-tuned by allowing selection on opposing factors that individually channel separate, upstream influences on polyphenism. It is also possible that gene duplications, as observed in $P$. exspectatus, could result from gene amplification in such a system. In such a case, fixation of duplicates and the specialisation of new developmental regulators would thereafter be possible ${ }^{33}$. Although functional assays have revealed only limited polyphenism function for paralogues of eud-1 $1^{34,35}$, the more extensive radiation of seud-1 homologues allows a better test of this idea. By revealing the precise factors controlling a polyphenism switch, it is now possible to reconstruct what changes to a switch have occurred across species that have diverged in their sensitivity to environmental pressures.

In conclusion, we have identified the genetic regulatory points that establish a threshold between plastic developmental phenotypes, and we have shown that modifications to these regulatory points correlate with divergent morph-ratios between species. Importantly, this model offers a foundation for identifying the genetic modifications that have occurred in other instances of polyphenism evolution, thereby revealing its generalisable features. The nematode family (Diplogastridae) that includes Pristionchus has many other species with this ancestral polyphenism, and among lineages there is a diversity of plasticity responses as well as plastic morphologies ${ }^{12,36}$. With our genetic description of a switch apparatus, it is possible to comparatively explore how evolutionary modifications to this switch-in regulation, function, or downstream targets-have facilitated a dramatic radiation of polyphenism-governed traits.

\footnotetext{
Methods

Nematode cultures. All Pristionchus strains were maintained on 6-cm Petri plates containing nematode growth medium (NGM) agar seeded with $300 \mu \mathrm{l}$ of Escherichia coli grown in L-broth. All cultures were maintained at $20-25^{\circ} \mathrm{C}$.

Forward genetics screen. Mutagenesis of the $P$. pacificus null mutant eud-1 (tu445) followed a described protcol ${ }^{37}$ : self-fertilising hermaphrodites $\left(\mathrm{P}_{0}\right)$ were mutagenised at the J4 (pre-adult) stage by incubation in $47 \mathrm{mM}$ ethyl methanesulfonate at $20^{\circ} \mathrm{C}$ for $3.5 \mathrm{~h}$ on a rocker at low speed (40 UPM), washed three times with $5 \mathrm{ml}$ of M9 buffer, transferred onto individual 10-cm NGM plates, and allowed to produce $\sim 20$ offspring each. These $\mathrm{F}_{1}$ self-fertilised to produce $\mathrm{F}_{2}$ on the same plates, and once most $F_{2}$ had reached the adult stage, we screened for nematodes of the Eu morph, which suppressed the Eud (all-St) phenotype. Eu $\mathrm{F}_{2}$ were cloned into individual culture lines. Phenotypes of $>100 \mathrm{~F}_{3}$ clones of each line were screened to confirm the mutant phenotype to be both homozygous and recessive. Pairwise complementation tests were performed for the seven isolated recessive mutants, and these tests grouped alleles $i u b 7$ and $i u b 8$. These two mutants were then backcrossed to the eud-1(tu445) strain four and six times, respectively, using the following strategy: for two rounds of backcrossing, mutant hermaphrodites were first crossed to eud-1(tu445) males, after which $\mathrm{F}_{1}$ were backcrossed to the strain pdl-2(tu463); eud-1(tu445), a strain in which the parental line had been marked with a recessive "dumpy" mutation ${ }^{15} ; \mathrm{BC}_{1} \mathrm{~F}_{1}$ were then selfed to recover $\mathrm{Eu}$
} $\mathrm{BC}_{1} \mathrm{~F}_{2}$, which were cloned and then backcrossed further as above.
Phenotype scoring. Mouth phenotypes were scored as previously described ${ }^{11}$. In short, the Eu phenotype was determined by the presence of (i) a claw-shaped dorsa tooth, (ii) a large, hooked subventral tooth, and (iii) a mouth wider than deep; the St phenotype was determined by (i) a narrow, dorsoventrally symmetrical dorsal tooth, (ii) absence of a subventral tooth, and (iii) a mouth narrower than deep. Rare $(<0.5 \%)$ intermediates between the two morphs were excluded from phenotype counts. Phenotypes were scored using differential interference (DIC) microscopy using a Zeiss AxioScope, except in the $\mathrm{F}_{2}$ screen for mutants, for which a Zeiss Discovery V.20 stereoscope was used for higher throughput. At least 60 individuals, with one exception $(n=25)$, were scored for each genotype (Supplementary Tables 4, 6, 9).

Genomic resequencing for mutant identification. To prepare samples for whole genomic sequencing, nemtatodes from five agar plates of each strain were washed with M9 buffer in a $50-\mathrm{ml}$ conical tube. Nematodes were then washed with $0.9 \%$ $\mathrm{NaCl}$ with ampicillin $(50 \mu \mathrm{g} / \mathrm{ml})$ for $12 \mathrm{~h}$, after which the nematode pellet was collected and washed with $0.9 \% \mathrm{NaCl}$ with ampicillin $(50 \mu \mathrm{g} / \mathrm{ml})$ and chloramphenicol $(25 \mu \mathrm{g} / \mathrm{ml})$ for $12 \mathrm{~h}$. After centrifuging at $1300 \times \mathrm{g}$ for $4 \mathrm{~min}$, the nematode pellet was collected and genomic DNA was extracted using a MasterPure DNA Purification Kit (Epicentre) and was quantified using an Agilent TapeStation 2200. DNA libraries for sequencing were prepared using a Nextera kit, diluted to a concentration of $0.45 \mathrm{pM}$ in $0.1 \%$ EB-Tween, and pooled as 6-plex. The libraries were sequenced as 150-bp paired-end reads on an Illumina NextSeq 300 to a theoretical coverage of $24 \times$. Reads from raw sequencing data were mapped to the $P$. pacificus genome (version Hybrid1, www.pristionchus.org) using the Burrows Wheeler Alignment tool ${ }^{38}$, and variants were called using SAMtools ${ }^{39}$.

Identification of mutant lesions. With the lists of potential variants for $i u b 7$ and $i u b 8$, we identified the causal lesions as follows. First, we excluded all variants from each list that were shared with the resequenced genome of the mutagenised eud-1 (tu445) strain $^{3}$ and with the dumpy pdl-2(tu463) strain (voucher EJR1018) used to create the $p d l-2(t u 463)$; eud-1(tu445) strain for backcrossing. Variants in predicted coding sequences were categorised with respect to the Hybrid1 assembly and AUGUSTUS annotation for the genome of $P$. pacificus (www.pristionchus.org). From the intersecting lists of variants, we excluded SNPs manually determined to be artefactual using the Integrative Genomics Viewer ${ }^{40}$. Following the identification of the mutant locus as Contig20-aug8366.t1 from resequenced genomes, mutant lesions were verified by Sanger sequencing (Supplementary Table 3).

Outcrossing of the eud-1 mutation. To determine the phenotype of a null seud-1 mutant without a background eud-1 mutation, we outcrossed the mutant seud-1 (iub7); eud-1(iub8) to the wild-type reference ("California," PS312) strain of $P$. pacificus. For the $\mathrm{P}_{0}$ cross, we used males of the mutant strain and California hermaphrodites, so that $\mathrm{F}_{1}$ males would inherit the autosomal seud-1 mutation but not the X-linked eud-1 mutation. $\mathrm{F}_{1}$ males were then outcrossed again to California hermaphrodites, virgin $\left(\mathrm{BC}_{1}\right)$ offspring of which were let to self-cross to re-segregate mutant seud-1 alleles from putative heterozygotes. Multiple $\mathrm{BC}_{1} \mathrm{~F}_{2}$ lines were cloned and $>200$ individuals per line were screened to confirm their all-Eu phenotype. Finally, the presence of the identified seud-1(iub7) lesion and the absence of the eud-1(tu445) lesion were additionally confirmed by PCR and Sanger sequencing (Supplementary Table 3).

Over-expression of seud-1 by genetic transformation. Transgenic $P$. pacificus nematodes were generated by microinjection of the gonads (germline) of adult hermaphrodites, which were then selfed, following which offspring with a reporter gene were screened among the $\mathrm{F}_{1}{ }^{41}$. To rescue function of and over-express seud-1, ovaries of outcrossed seud-1(iub7) mutants were injected with an 11-kb genomic clone containing the putative $3.9-\mathrm{kb}$ promoter region of wild-type seud-1 and a 6.7 $\mathrm{kb}$ coding sequence including its 330 -bp $3^{\prime}$ regulatory region $(10 \mathrm{ng} / \mu \mathrm{l})$. This construct was delivered with the co-injection marker egl-20 promoter::TurboRFP $(10 \mathrm{ng} / \mu \mathrm{l})$ and digested genomic carrier DNA $(60 \mathrm{ng} / \mu \mathrm{l})$ from the recipient strain. To over-express $n h r-40$ in seud-1 mutants, an extrachromosomal array from a $n h r-40$ rescue line generated previously ${ }^{15}$ was crossed into the outcrossed seud-1 (iub7) strain.

Crosses to study effects of relative gene dosage. Genetic crossing experiments were performed to test for haploinsufficiency and opposing dosages between eud-1 and seud-1. In our cross panel, all possible genotype combinations (i.e., homozygotes, heterozygotes, and hemizygotes) of wild-type (California) and mutant (tu445 and iub7) alleles of eud-1 and seud-1 were created. The backcrossed, doublemutant line seud-1(iub7); eud-1(tu445) (voucher EJR1029), the outcrossed line seud-1(iub7) (voucher EJR1039), the backcrossed ( $\times 6)$ parental line eud-1(tu445), and the California strain were used for creating these genotypes. To correctly identify $F_{1}$ instead of self-offspring from these crosses, all polyphenism mutants were marked with the recessive dumpy marker $p d l-2(t u 463)$, such that crossoffspring could be easily distinguished by the rescue of a non-dumpy phenotype. In addition to using previously generated lines ${ }^{15}$, we created the double mutant seud-1 (iub7); pdl-2(tu463) and triple mutant seud-1(iub7); pdl-2(tu463); eud-1(tu445). 
Homozygosity of mutations was confirmed by Sanger sequencing of all alleles in $>10$ clones per line (Supplementary Table 3 ).

Localisation of seud-1 expression. We localised expression of seud-1 in P. pacificus using a transcriptional reporter. To express this reporter, we transformed $P$. pacificus germline cells along with co-injection markers as described above. Because seud-1 is dosage-dependent, we predicted its expression in the California strain to be low, as shown previously for the St-promoting $n h r-40^{15}$. Therefore, we used $P$. pacificus strain RS5200B for transformation, as this strain is a natural variant that is highly St in laboratory culture ${ }^{3}$ and was thus more likely than the California strain to express seud-1 at detectable levels. The construct used for genetic transformation was ligated from the following sequence fragments: (i) the $4.2-\mathrm{kb}$ putative promoter region of seud-1, (ii) a coding sequence consisting of a start codon, a nuclear localisation signal, and TurboRFP, and (iii) the $3^{\prime}$ untranslated region of the gene Ppa-rpl-23. The construct was cloned into pCR4 TOPO vector and maintained in a TOP10 E. coli clone, which served as its source for microinjection experiments. Two independent transgenic lines were generated, and 10 animals at each life-stage (for adults, $5 \mathrm{St}$ and $5 \mathrm{Eu}$ ) were examined using a Zeiss AxioImager.

Environmentally altered eud-1 and seud-1 expression. Raw RNA reads, which were produced by nematodes raised on E. coli OP50, Cryptococcus albicans, and C. curvatus $^{22}$, were downloaded from the NCBI database (accession number: SRP081198). The sequence published as UMM-S71-39.9-mRNA-1 was identified as seud-1, and UMM-S2877-2.55-mRNA-1 as eud-1. Paired-end reads ( $2 \times 150 \mathrm{bp})$ from two biological replicates for each condition were aligned to the P. pacificus reference genome (version El Paco, www.pristionchus.org) using STAR (version 2.5.3 $)^{42}$ under default settings. Read counts were achieved using the function FeatureCounts in Rsubread Bioconductor package (version 1.24.2) ${ }^{43}$ and genes with a low number count (i.e., low-expressed) were filtered out. Reads mapped to seud-1 and eud-1 genes were examined manually in IGV viewer (version 2.3.77) ${ }^{40}$ to eliminate false mapping. Differential expression analysis was performed in edge $\mathrm{R}^{44}$, with $p$-values adjusted according to the Benjamini-Hochberg procedure to restrict false discovery rate.

Phylogenetic analysis. Evolutionary history of seud-1 homologues in Pristionchus and outgroups was inferred from predicted homologues mined from available nematode genomes using the criterion of reciprocal best BLAST similarity with C. elegans ssu-1. For reconstruction of the phylogenetic history of seud-1, predicted amino-acid sequences encoded by identified ssu- 1 homologues were aligned using the E-INS-I algorithm and default settings in MAFFT (version 7.1) ${ }^{45}$. The gene tree of predicted SSU-1 amino-acid sequences was inferred under the maximum likelihood (ML) criterion, as implemented in RAxML (version 8$)^{46}$. Fifty independent analyses were performed. Analyses invoked a Whelan and Goldman model with a gamma-shaped distribution of rates across sites. Bootstrap support for the most likely tree among all runs was estimated by 500 pseudoreplicates.

Selection analyses of seud-1 duplicates. We tested for purifying selection among seud-1 alleles using a pairwise counting method of $d_{\mathrm{N}} / d_{\mathrm{S}}^{47}$, as implemented in the programme PAML ${ }^{48}$. We performed three comparisons: (i) Pex-seud-1.1 to Pex-seud-1.2; (ii) Pex-seud-1.1 to Ppa-seud-1(California); and (iii) Pex-seud-1.2 to Ppa-seud-1(California).

Hybrid crosses to study effects of gene duplication. Hybrid crosses followed the same logic described for studying relative gene dosage in $P$. pacificus, with the following modifications. In crosses of $P$. pacificus males to $P$. exspectatus females, a phenotypic (e.g., dumpy) marker was not used or needed, as $P$. exspectatus requires sexual reproduction to produce offspring. Also, only female hybrids were used to score phenotypes, as male $\mathrm{F}_{1}$ from reciprocal crosses have different genetic backgrounds that were previously found to have asymmetrical epistatic effects on the mouth polyphenism ${ }^{3}$.

seud-1 and eud-1 expression in natural polyphenism variants. To quantify transcripts from the California strain and two St-biased strains (RS5200B and RS5410), we used J3 and J4 nematodes from each of these populations. To collect nematodes mostly at these stages, cultures were synchronised by starting with eggs released by bleaching gravid mothers ${ }^{49}, 48 \mathrm{~h}$ after which nematodes were washed from plates and pooled. Following centrifugation of collected nematodes at $1300 \times g$ for $4 \mathrm{~min}$, nematode pellets were resuspended in $1 \mathrm{ml}$ of TRIzol. For RT-PCR, total RNA was extracted from the homogenised solutions of nematodes in TRIzol using the Zymo Direct-zol RNA Miniprep kit (Zymo Research). $1 \mu \mathrm{g}$ of total RNA was used for first-strand cDNA synthesis with random hexamers in a $20-\mu l$ reaction using the SuperScript III Reverse Transcriptase kit (Invitrogen). cDNA was diluted five times, and $1.5 \mu \mathrm{l}$ of the final volume was used for a $10 \mu \mathrm{l}$ PCR. Real-time PCR to quantify expression was performed on a Roche LightCycler 96 system, using SYBR GREEN reaction mix and the manufacturer's (Roche) software and with the genes encoding beta-tubulin ( $t b b-4)$ and an iron-binding protein (Y45F10D) as the reference genes ${ }^{50}$. Primers for RT-qPCR of P. pacificus seud- 1 and eud- 1 are listed in Supplementary Table 10 . The PCR cycle was: $10 \mathrm{~min}$ at $95^{\circ} \mathrm{C}$, followed by 45 cycles of $10 \mathrm{~s}$ at $95^{\circ} \mathrm{C}, 20 \mathrm{~s}$ at $52^{\circ} \mathrm{C}$, and $15 \mathrm{~s}$ at $72^{\circ} \mathrm{C}$, with a single fluorescence read at the end of each extension. Each PCR reaction was performed on three independent biological replicates and three technical replicates for each group. Melting-curve analysis and gel electrophoresis were performed to ensure the absence of non-specific products or primer dimerisation, and PCR efficiency was identified with a 5-log titration of pooled cDNA. Relative expression levels were determined using the $\Delta \Delta \mathrm{Ct}$ method $^{50}$, with California strain designated as the control group.

Genetic inbreeding of $\boldsymbol{P}$. exspectatus polyphenism variants. For systematic inbreeding to fix different plasticity phenotypes, we started with a source population of $P$. exspectatus (RS5522) previously shown to harbour heterozygosity in genes affecting the mouth-morph ratio ${ }^{3}$. This population was recovered from a frozen voucher of a culture that, prior to freezing, had been established from multiple individuals collected from a vector beetle ${ }^{23}$ and had been kept in laboratory culture for about 1 year on large-diameter $(13 \mathrm{~cm})$ plates, with subcultures established through "chunking" 51 , whereby dozens of individuals were transferred. This voucher comprised nematodes from $~ 10$ separate cultures maintained under the conditions above and frozen according to standard proto$\mathrm{cols}^{37}$. This population was the parent source of the inbred strain RS5522B, in which a Eud phenotype had previously been fixed through inbreeding ${ }^{3}$. From the revived RS5522 strain, we systematically inbred two new lines for 10 generations each, using a single male-female pair at each generation. Because systematic inbreeding of a dioecious, or obligately outcrossing, species was likely to lead to negative fitness effects on the populations, 10 individual pairs were mated at each generation, with the subsequent generation being established from the most fecund pair; furthermore, all pairs from the previous four generations were kept stable at low temperature $\left(6^{\circ} \mathrm{C}\right)$ as insurance in case lines from selected pairs suffered inbreeding generations later. All crosses were performed under standardised environmental conditions as described for experimental crosses above. As a result of this inbreeding method, we were able to establish lines, which were in turn frozen as vouchers that were both inbred and without obvious negative effects on brood size and generation time. Additionally, because the thawed voucher was highly St-biased, we promoted the retention of the polyphenism (i.e., as opposed to a fixed Eud phenotype), by selecting for the Eu morph for two generations. Specifically, males and females selected for breeding during the first two generations were both $\mathrm{Eu}$, whereas in subsequent generations inbreeding was random with respect to mouth phenotype. The variable morph-ratio phenotypes of the resulting inbred lines (EJR16 and EJR18) were then recorded under a standardised environment as described above.

seud-1 and eud-1 expression in inbred polyphenism variants. To quantify transcripts from inbred $P$. exspectatus lines, mixed-stage nematodes were collected and prepared for quantitative real-time PCR (RT-qPCR) as described above for expression quantification in natural polyphenism variants. Because the coding sequences of Pex-seud-1.1 and Pex-seud-1.2 are highly similar, primers matching both genes were used to amplify the total number of Pex-seud-1 transcripts. Two non-overlapping pairs of primers were used, and transcripts of both Pex-seud-1.1 and Pex-seud-1.2 were confirmed by manual inspection of trace files of sequences for both RT-qPCR amplicons. RT-qPCR followed the procedure described above for the quantification of $P$. pacificus variants, with $t b b-4$ (encoding beta-tubulin) used as the reference gene ${ }^{50}$. Relative expression levels were determined with line EJR16 designated as the control group. To quantify seud-1 expression, two primer sets were used and all data from two separate runs with the set "seud-1A" (Supplementary Table 8) and one run with the set "seud-1B" were combined. PCR products were purified and submitted to Sanger Sequencing (Eurofins Genomics LLC) to confirm the presence of both duplicate genes. eud-1 expression was quantified from two separate runs using eud-1 primers (Supplementary Table 8).

Statistical analyses. All statistical analyses were conducted in R (version 3.4.3) ${ }^{52}$ Polyphenism phenotypes were recorded as proportional data, specifically the percentage of Eu per total number of individuals screened. To determine whether there were differences in morph ratios, we used generalised linear models with binomial error and a logit link function, designating genotype as the explanatory variable. Significance was assessed for (i) epistatic interactions of $n h r-40$ and seud-1 (Fig. 2a); (ii) genotypes from hybrid crosses; (iii) differences between P. exspectatus inbred lines (Fig. 4d). Replicates within lines were included in each model, and significance of differences was determined using a $\chi^{2}$-test for pairwise comparisons, and a $Z$-test where multiple comparisons were made. Significant differences between specific contrasts (i.e., between results of hybrid crosses) in the final models were determined using post-hoc Tukey's honest significant difference tests as implemented in the package lsmeans ${ }^{53}$. For graphical representation of phenotype data, mouth-morph frequencies were pooled across replicates within lines, with $95 \%$ confidence intervals estimated using a binomial test; these results are qualitatively identical to those derived from the generalised linear model.

Significance of differences in expression of eud-1 and seud-1 transcripts among wild isolates of $P$. pacificus and between inbred $P$. exspectatus lines was determined by a linear mixed model, which accounted for repeated measures within biological replicates, using the lme function in the nlme package ${ }^{54}$. Log fold change was used 
as the response variable, strain as a fixed explanatory variable, and biological replicate as a random variable. For tests involving $P$. exspectatus, this method also confirmed that there were no differences among experimental runs (i.e., different primer pairs) for quantification of seud-1 expression.

\section{Data availability}

Gene sequences original to this study ( $P$. arcanus) and raw phenotypic data have been deposited in the Figshare repository under https://figshare.com/articles/ Bui_Ivers_Ragsdale-deposit_xlsx/6708674.

Received: 27 February 2018 Accepted: 11 July 2018

Published online: 08 October 2018

\section{References}

1. West-Eberhard, M. J. Developmental Plasticity and Evolution (Oxford University Press, Oxford, UK, 2003).

2. Moczek, A. P. et al. The role of developmental plasticity in evolutionary innovation. Proc. R. Soc. Lond. B 278, 2705-2713 (2011)

3. Ragsdale, E. J., Müller, M. R., Rödelsperger, C. \& Sommer, R. J. A developmental switch coupled to the evolution of plasticity acts through a sulfatase. Cell 155, 922-933 (2013).

4. Kijimoto, T., Moczek, A. P. \& Andrews, J. Diversification of doublesex function underlies morph-, sex-, and species-specific development of beetle horns. Proc. Natl Acad. Sci. USA 109, 20526-20531 (2012).

5. Xu, H. J. et al. Two insulin receptors determine alternative wing morphs in planthoppers. Nature 519, 464-467 (2015).

6. Klein, A., et al. Evolution of social insect polyphenism facilitated by the sex differentiation cascade. PLoS Genet. 12, e1005952 (2016)

7. Brakefield, P. M., Kesbeke, F. \& Koch, P. B. The regulation of phenotypic plasticity of eyespots in the butterfly Bicyclus anynana. Am. Nat. 152, 853-860 (1998).

8. Suzuki, Y. \& Nijhout, H. F. Evolution of a polyphenism by genetic accommodation. Science 311, 650-652 (2006).

9. Serobyan, V., Ragsdale, E. J. \& Sommer, R. J. Adaptive value of a predatory mouth-form in a dimorphic nematode. Proc. R. Soc. B 281, 20141334 (2014).

10. Bento, G., Ogawa, A. \& Sommer, R. J. Co-option of the hormone-signalling module dafachronic acid-DAF-12 in nematode evolution. Nature $\mathbf{4 6 6}$, 494-497 (2010).

11. Serobyan, V., Ragsdale, E. J., Müller, M. R. \& Sommer, R. J. Feeding plasticity in the nematode pristionchus pacificus is influenced by sex and social context and is linked to developmental speed. Evol. Dev. 15, 161-170 (2013).

12. Susoy, V., Ragsdale, E. J., Kanzaki, N. \& Sommer, R. J. Rapid diversification associated with a macroevolutionary pulse of developmental plasticity. eLife $\mathbf{4}$, e05463 (2015).

13. Bose, N. et al. Complex small-molecule architectures regulate phenotypic plasticity in a nematode. Angew. Chem. 51, 12438-12443 (2012).

14. Serobyan, V. et al. Chromatin remodelling and antisense-mediated upregulation of the developmental switch gene eud-1 control predatory feeding plasticity. Nat. Commun. 7, 12337 (2016).

15. Kieninger, $M$. R. et al. The nuclear hormone receptor NHR-40 acts downstream of the sulfatase EUD-1 as part of a developmental plasticity switch in Pristionchus. Curr. Biol. 26, 2174-2179 (2016).

16. Carroll, B. T., Dubyak, G. R., Sedensky, M. M. \& Morgan, P. G. Sulfated signal from ASJ sensory neurons modulates stomatin-dependent coordination in Caenorhabditis elegans. J. Biol. Chem. 281, 35989-35996 (2006).

17. Hattori, K., Inoue, M., Inoue, T., Arai, H. \& Tamura, H. O. A novel sulfotransferase abundantly expressed in the dauer larvae of Caenorhabditis elegans. J. Biochem. 139, 355-362 (2006).

18. Bumbarger, D. J., Riebesell, M., Rodelsperger, C. \& Sommer, R. J. System-wide rewiring underlies behavioral differences in predatory and bacterial-feeding nematodes. Cell 152, 109-119 (2013).

19. White, J. The anatomy. In The Nematode Caenorhabditis elegans (ed. Wood, W. B.) 17, 81-122 (Cold Spring Harbor Laboratory Press, Cold Spring Harbor, US, 1988).

20. Wright, K. A. \& Thomson, J. N. The buccal capsule of Caenorhabditis elegans (Nematoda: Rhabditoidea): an ultrasatructural study. Can. J. Zool. 59, 1952-1961 (1981)

21. Baldwin, J. G. et al. The buccal capsule of Aduncospiculum halicti (Nemata: Diplogasterina): an ultrastructural and molecular phylogenetic study. Can. J. Zool. 75, 407-423 (1997)

22. Sanghvi, G. V. et al. Life history responses and gene expression profiles of the nematode Pristionchus pacificus cultured on Cryptococcus yeasts. PLoS ONE 11, 0164881 (2016).

23. Kanzaki, N., Ragsdale, E. J., Herrmann, M., Mayer, W. E. \& Sommer, R. J. Description of three Pristionchus species (Nematoda: Diplogastridae) from
Japan that form a cryptic species complex with the model organism P. pacificus. Zool. Sci. 29, 403-417 (2012).

24. Morgan, K. et al. Multi locus analysis of Pristionchus pacificus on La Reunion Island reveals an evolutionary history shaped by multiple introductions, constrained dispersal events and rare out-crossing. Mol. Ecol. 21, 250-266 (2012).

25. Rödelsperger, C. et al. Characterization of genetic diversity in the nematode Pristionchus pacificus from population-scale resequencing data. Genetics 196, 1153-1165 (2014)

26. Nijhout, H. F. Development and evolution of adaptive polyphenisms. Evol. Dev. 5, 9-18 (2003).

27. Mueller, J. W., Gilligan, L. C., Idkowiak, J., Arlt, W. \& Foster, P. A. The regulation of steroid action by sulfation and desulfation. Endocr. Rev. 36, 526-563 (2015)

28. Shi, X. et al. Cholesterol sulfate and cholesterol sulfotransferase inhibit gluconeogenesis by targeting hepatocyte nuclear factor 4. Mol. Cell Biol. 34, 485-497 (2014).

29. Ketterson, E. D. \& Nolan, Jr,V. Adaptation, exaptation, and constraint: a hormonal perspective. Am. Nat. 154, S4-S25 (1999).

30. Zijlstra, W. G., Steigenga, M. J., Koch, P. B., Zwaan, B. J. \& Brakefield, P. M Butterfly selected lines explore the hormonal basis of interactions between life histories and morphology. Am. Nat. 163, E76-E87 (2004).

31. Hoekstra, H. E., Hirschmann, R. J., Bundey, R. A., Insel, P. A. \& Crossland, J. P. A single amino acid mutation contributes to adaptive beach mouse color pattern. Science 313, 101-104 (2006).

32. Brožová, E., Šimecková, K., Kostrouch, Z., Rall, J. E. \& Kostrouchová, M. NHR-40, a Caenorhabditis elegans supplementary nuclear receptor, regulates embryonic and early larval development. Mech. Dev. 123, 689-701 (2006).

33. Ohno, S. Evolution by Gene Duplication (Springer-Verlag, New York, Stuttgart US, 1970).

34. Ragsdale, E. J. \& Ivers, N. A. Specialization of a polyphenism switch gene following serial duplications in Pristionchus nematodes. Evolution 70, 2155-2166 (2016).

35. Sieriebriennikov, B. et al. A developmental switch generating phenotypic plasticity is part of a conserved multi-gene locus. Cell Rep. 23, 2835-2843 (2018).

36. Susoy, V. et al. Large-scale diversification without genetic isolation in nematode symbionts of fig. Sci. Adv. 2, e1501031 (2016).

37. Pires da Silva, A. Pristionchus pacificus genetic protocols. WormBook https:// doi.org/10.1895/wormbook.1.114.1(2006).

38. Li, H. \& Durbin, R. Fast and accurate short read alignment with BurrowsWheeler transform. Bioinformatics 25, 1754-1760 (2009).

39. Li, H. et al. The sequence alignment/map format and SAMtools. Bioinformatics 25, 2078-2079 (2009).

40. Thorvaldsdóttir, H., Robinson, J. T. \& Mesirov, J. P. Integrative Genomics Viewer (IGV): high-performance genomics data visualization and exploration. Brief. Bioinform. 14, 178-192 (2013).

41. Schlager, B., Wang, X., Braach, G. \& Sommer, R. J. Molecular cloning of a dominant roller mutant and establishment of DNA-mediated transformation in the nematode Pristionchus pacificus. Genesis 47, 300-304 (2009).

42. Dobin, A. et al. STAR: ultrafast universal RNA-seq aligner. Bioinformatics 29, 15-21 (2013)

43. Liao, Y., Smyth, G.K. \& Shi, W. The Subread aligner: fast, accurate and scalable read mapping by seed-and-vote. Nucleic Acids Res. 41, e108 (2013).

44. Robinson, M. D., McCarthy, D. J. \& Smyth, G. K. edgeR: a Bioconductor package for differential expression analysis of digital gene expression data. Bioinformatics 26, 139-140 (2010).

45. Katoh, K., Kuma, K., Toh, H. \& Miyata, T. MAFFT version 5: improvement in accuracy of multiple sequence alignment. Nucleic Acids Res. 33, 511-518 (2005).

46. Stamatakis, A. RAxML version 8: a tool for phylogenetic analysis and postanalysis of large phylogenies. Bioinformatics 30, 1312-1313 (2014).

47. Yang, Z. \& Nielsen, R. Estimating synonymous and nonsynonymous substitution rates under realistic evolutionary models. Mol. Biol. Evol. 17, $32-43(2000)$.

48. Yang, Z. PAML 4: phylogenetic analysis by maximum likelihood. Mol. Biol. Evol. 24, 1586-1591 (2007).

49. Hope, I. A. C. elegans: A Practical Approach. (Oxford University Press, Oxford, UK, 1999).

50. Schuster, L. N. \& Sommer, R. J. Expressional and functional variation of horizontally acquired cellulases in the nematode Pristionchus pacificus. Gene 506, 274-282 (2012).

51. Stiernagle, T. Maintenance of C. elegans. WormBook 11, 1-11, https://doi.org/ 10.1895/wormbook.1.101.1 (2006).

52. R Core Team. R: A Language and Environment for Statistical Computing. 55, 275-286 (R Foundation for Statistical Computing, Vienna, Austria, 2015).

53. Lenth, R. V. Least-squares means: the R package lsmeans. J. Stat. Softw. 69 , $1-33(2016)$ 
54. Pinheiro, J., Bates, D., DebRoy, S. \& Sarkar, D. nlme: linear and nonlinear mixed effects models. R package v. 3.1-127 (2016)

55. Bargmann, C. Chemosensation in C. elegans. WormBook https://doi.org/ 10.1895/wormbook.1.123.1 (2006).

56. Hu, P. J. Dauer. WormBook https://doi.org/10.1895/wormbook.1.144.1 (2007).

\section{Acknowledgements}

We thank the Indiana University Center for Genomics and Bioinformatics for DNA library preparation and genomic resequencing, Susan Feldt and Ryan Mueller for their help with nematode crosses, R. Taylor Raborn for suggestions on transcriptomic analyses, and Meagan Pritchard for media preparation and strain keeping. We also thank Ralf Sommer and Christian Rödelsperger (Max Planck Institute for Developmental Biology) for sharing their unpublished genome sequence of $P$. arcanus. This work was funded by the National Science Foundation (IOS 1557873).

\section{Author contributions}

E.J.R. conceived and designed the study; L.T.B. and N.A.I. conducted the experiments; L. T.B., N.A.I., and E.J.R. analysed the data; L.T.B., N.A.I., and E.J.R. wrote the manuscript.

\section{Additional information}

Supplementary Information accompanies this paper at https://doi.org/10.1038/s41467018-05612-8.
Competing interests: The authors declare no competing interests.

Reprints and permission information is available online at http://npg.nature.com/ reprintsandpermissions/

Publisher's note: Springer Nature remains neutral with regard to jurisdictional claims in published maps and institutional affiliations.

(c) (i) Open Access This article is licensed under a Creative Commons Attribution 4.0 International License, which permits use, sharing, adaptation, distribution and reproduction in any medium or format, as long as you give appropriate credit to the original author(s) and the source, provide a link to the Creative Commons license, and indicate if changes were made. The images or other third party material in this article are included in the article's Creative Commons license, unless indicated otherwise in a credit line to the material. If material is not included in the article's Creative Commons license and your intended use is not permitted by statutory regulation or exceeds the permitted use, you will need to obtain permission directly from the copyright holder. To view a copy of this license, visit http://creativecommons.org/ licenses/by/4.0/.

(C) The Author(s) 2018 